\title{
Parasitoses intestinais e fatores de risco associados em crianças em um município do Nordeste Brasileiro
}

\author{
Intestinal parasites and risk factors associated $f$ in children in a Brazilian Northeast \\ municipality
}

\author{
Jairina Nunes Chaves ${ }^{1 *}$, Gizelia Araújo Cunha², Tharliane Silva Chaves ${ }^{3}$, Anderson Araújo Corrêa ${ }^{4}$, \\ Ewaldo Eder Carvalho Santana ${ }^{5}$, Rita de Maria Seabra Nogueira ${ }^{6}$
}

\begin{abstract}
${ }^{1}$ Mestre em Biodiversidade, Ambiente e Saúde pela Universidade Estadual do Maranhão (UEMA), Especialista em Saúde Pública e da Família pela Faculdade de Ensino Superior Dom Bosco, Professora na Rede Municipal de Ensino de Caxias, MA; ${ }^{2}$ Mestre em Biodiversidade, Ambiente e Saúde pela UEMA, Professora na Universidade

Federal do Maranhão (UFMA); ${ }^{3}$ Mestre em Biodiversidade, Ambiente e Saúde pela UEMA; ${ }^{4}$ Mestre em Biodiversidade, Ambiente e Saúde pela UEMA, Enfermeiro vinculado ao Governo do Estado do Amazonas; ${ }^{5}$ Doutor em Engenharia Elétrica pela Universidade Federal de Campina Grande (UFCG), Professor da UEMA; ${ }^{6}$ Médica Veterinária, Doutora em Ciências pela Fundação Oswaldo Cruz (FIOCRUZ/RJ), Professora da UEMA
\end{abstract}

\begin{abstract}
Resumo
Introdução: os parasitos gastrintestinais são causas de morbidade em crianças, podendo determinar quadros de anemia e diarréria. Podem estar associados as condições socioeconômicas, de saneamento básico e práticas de higiene. Objetivo: o objetivo desta investigação foi identificar as parasitoses intestinais e os fatores de risco associados em crianças menores de 12 anos cadastradas em duas Unidades Básicas de Saúde do município de Caxias, estado do Maranhão. Metodologia: neste estudo transversal 422 exames coproparasitológicos pela técnica de Hoffman, Pons e Janner foram realizados. Para comparar as proporções utilizou-se o teste Qui-quadrado ou teste exato de Fisher. Empregou-se análises univariadas e multivariadas por regressão logística para avaliar a associação entre a infecção por enteroparasitos e os fatores de risco. Resultados: a frequência de parasitos intestinais foi $49,05 \%$, sendo predominante o Ascaris lumbricoides (68,18\%) e a Entamoeba sp. (65,38\%). Conforme a análise estatística, ingerir água sem tratamento domiciliar e não lavar as mãos antes das refeições podem ser considerados como fatores de risco para as enteropatias parasitárias. Conclusão: ressalta-se a necessidade de implementar políticas públicas que visem reduzir as altas taxas destes parasitos, e que contribuam para melhorar a qualidade de vida dessa população.
\end{abstract}

Palavras-chave: Parasitoses. Fatores de risco. Crianças.

\begin{abstract}
Introduction: intestinal parasites are cause of morbidity in children and can determine anemia and diarrhea. It can be associated to social economical conditions, basic sanitation and hygiene. Objective: the objective of this research was to identify tne intestinal parasitosis and the risk factors associated in children less than 12 years old registered in two Basic Health Units in Caxias municipality, state of Maranhão. Metodology: in this cross-sectional study 422 fecal exams were performed by the technique of Hoffman, Pons and Janner in 422 children. To compare the proportions, the chi-square test or Fisher's exact test was used. Univariate and multivariate analyzes were used by logistic regression to evaluate the association between enteroparasite infection and risk factors. Results: the frequency of intestinal parasites was 49.05\%, being predominant Ascaris lumbricoides (68.8\%) and Entamoeba sp. (65.38\%). According to statistical analysis, drinking water without home treatment and not washing hands before meals, can be considered as risk factors for parasitic enteropathies. Conclusion: It's necessary to implement public policies aimed at reducing the high rates of intestinal parasites, which contributes to improve the quality of life of this population.
\end{abstract}

Keywords: Parasitosis. Risk factors. Children.

\section{INTRODUÇÃO}

As infecções por parasitos intestinais podem determinar diarréia, anemia e desnutrição com consequente impacto físico e intelectual, notadamente em crianças em idade escolar ${ }^{1}$. Segundo dados do Ministério da Saúde, ocorreram no ano de 2016 um total de 36.350 óbi-

Correspondente/Corresponding: *Jairina Nunes Chaves - End: Rua da Fazenda, $n^{\circ} 23$, Quadra A, Alto do Ipem, Bairro Antenor Viana, CEP: 65603-310, Caxias, Maranhão - Tel: (99) 98197-6231 - E-mail: inanunes@hotmail.com tos infantis, sendo que as doenças parasitárias ocupam a quarta posição no número de mortes relacionadas².

Resultados de estudos sobre a prevalência de parasitoses intestinais demonstram relação entre a ocorrência dessas enfermidades e as condições socioeconômicas, saneamento e práticas de higiene ${ }^{3-5}$.

No Brasil, as parasitoses intestinais são rotineiramente tratadas na Atenção Primária à Saúde, a qual se constitui por um conjunto de ações no âmbito individual e coletivo, voltadas para a promoção, proteção da saúde, prevenção de agravos, diagnóstico, trata- 
mento, reabilitação e redução de danos. Essa política visa desenvolver uma atenção integral que impacte na situação de saúde e autonomia dos cidadãos, bem como nos determinantes e condicionantes de saúde da coletividade ${ }^{6}$.

Dessa forma, a Estratégia Saúde da Família (ESF) assume papel fundamental no desenvolvimento de estratégias voltadas para o controle dessas doenças. A importância da ESF para o enfrentamento das enteropatias parasitárias está relacionada ao desenvolvimento de ações de educação em saúde direcionadas para a comunidade e para os grupos de riscos, realização de visitas domiciliares pela equipe multiprofissional, marcação de consultas para o diagnóstico e tratamento, além da realização de vigilância epidemiológica ${ }^{7}$.

Este estudo teve como objetivo identificar os fatores de riscos associados à ocorrência de parasitoses intestinais em crianças menores de 12 anos cadastradas em duas Unidades Básicas de Saúde do município de Caxias, estado do Maranhão.

\section{METODOLOGIA}

\section{População amostral}

Trata-se de um estudo transversal de avaliação de ocorrência de parasitos intestinais em crianças menores de 12 anos cadastradas na área de abrangência de duas Unidades Básicas de Saúde (UBS) do município Caxias, situada no leste do estado do Maranhão, na região do Médio Itapecuru. O município ocupava a 28a posição com Índice de Desenvolvimento Humano Municipal (IDHM) de 0,624, em 2010, sendo situado na faixa de desenvolvimento humano médio, entre 0,600 e 0,6998.

O município de Caxias possui 36 UBS, sendo 25 localizadas na zona urbana e 11 na zona rural. Dentre essas unidades de saúde foram selecionadas duas para o desenvolvimento do estudo, UBS Antenor Viana, localizada na zona urbana, e UBS Santo Antonio, pertencente a zona rural.

A UBS Antenor Viana está localizada no bairro de mesmo nome. A área de abrangência deste estabelecimento de saúde compreende as residências localizadas nos bairros Antenor Viana, Seriema e Teso Duro. É oportuno relatar, que essa USB também possui áreas anexas, as quais estão localizadas na zona rural. Contudo, estas foram excluídas do estudo. Assim, a área que fez parte da pesquisa possui duas equipes de ESF, com um total de 1.076 famílias cadastradas.

A UBS Santo Antônio, zona rural, fica localizada no povoado de mesmo nome, 20 Distrito do município de Caxias. Fazem parte da área de cobertura dessa Unidade de Saúde várias localidades. A coleta de dados ocorreu nos povoados Alecrim, Mucambo, Boca da Mata e Santo Antônio. Esses locais foram selecionados em decorrência da grande quantidade de famílias cadastradas.
A UBS supracitada tem um total de 575 famílias cadastradas. Contudo, segundo informações da Coordenação de Atenção Básica, essa área não é 100\% coberta pela ESF, devido ao número insuficiente de Agentes Comunitários de Saúde (ACS).

A população fonte do estudo compreendeu o conjunto de 1.149 crianças elegíveis. Destas, 747 residem nas microáreas da UBS Antenor Viana e 402 nas microáreas da UBS Santo Antônio. Para calcular a amostra, a população foi dividida em estrato por área do município, rural e urbana. Dessa forma, o tamanho amostral estimado foi de 422 crianças, sendo 235 cadastradas na UBS Antenor Viana e 187 na UBS Santo Antônio, considerando-se nível de confiança de $95 \%$, prevalência de $33,1 \%$ e margem de erro de $5 \%$.

Considerando que no Brasil não existe uma prevalência geral para infecções parasitárias, utilizou-se para o cálculo amostral a frequência de $33,1 \%$, obtida em estudo ocorrido no município de Chapadinha, estado do Maranhão9.

Inclui-se na pesquisa crianças menores de 12 anos cadastradas nas áreas de abrangências das UBS selecionadas. Foram excluídas as crianças cujos pais ou responsáveis não assinaram o Termo de Assentimento Livre e Esclarecido (TALE).

A coleta de dados ocorreu no período de janeiro a abril de 2017. As informações referentes aos indivíduos cadastrados na UBS Santo Antônio foram obtidas nas escolas municipais José Gonçalves Costa, Conselheiro Sinval, Eduvirgem Gonçalves Costa e Domingos da Silva, localizadas respectivamente nos povoados Alecrim, Mucambo, Santo Antônio e Boca da Mata, respectivamente. Quanto aos dados da zona urbana, a coleta foi realizada nas creches municipais Isabel Dolores Leão Brito e Elza de Sousa Fonseca, situadas nos bairros Antenor Viana e Seriema, respectivamente. Além disso, coletaram-se dados nas residências dos moradores do Povoado Alecrim e Mucambo, e no bairro Antenor Viana com intuito de conhecer a real situação de moradia e saneamento básico dos indivíduos incluídos no estudo.

\section{Coleta das amostras}

Após o preenchimento do formulário, foi entregue um frasco descartável (coletor universal) com conservante (formol a 10\%), devidamente identificado com nome da criança e do responsável, com instruções sobre a forma correta de coleta das fezes. $O$ recolhimento das amostras ocorreu nas escolas e nas residências cinco dias após a assinatura do TALE.

Os exames coproparasitológicos, para deteç̧ão de ovos de helmintos e cisto de protozoários foram realizados pelo método de sedimentação espontânea-técnica de Hoffman, Pons e Janner ${ }^{10}$. 


\section{Questionário sociodemográfico}

Para se conhecer o perfil sociodemográfico da população amostral foi aplicado um formulário contendo questões fechadas e abertas para pais ou responsáveis pelas crianças. Através do formulário, coletaram-se dados sobre a situação socioeconômica da família, fatores predisponentes à enteroparasitoses relacionados às condições de moradia, saneamento básico, hábitos alimentares, hábitos de higiene e contatos das crianças com animais domésticos.

\section{Análise estatística}

Para análise dos dados foi utilizado o programa Statistical Package for Social Sciences, version 20.0 for Windows (SPSS In. Chicago, II, USA). A comparação das proporções realizada por meio do teste Qui-quadrado $\left(X^{2}\right)$ ou teste exato de Fisher. Foram usadas análises univariadas e multivariadas por regressão logística para avaliar a associação entre a infecção por parasitoses intestinais e os prováveis fatores de riscos, considerando o cálculo do Odds Ration (OR), com intervalo de confiança (IC) de $95 \%$. Na análise multivariada, entraram no modelo aquelas variáveis que na análise univariada apresentaram $p$-valor $<0,20$. $O$ nível de significância estatística de $5 \%(p<0,05)$ foi considerado para todos os testes.

Nas análises estatísticas foi considerada como variável dependente a presença de enteroparasitos e as variáveis independentes os aspectos socioeconômicos, condições de moradia e saneamento básico, presença de animais domésticos, higiene e conhecimento dos familiares sobre parasitoses intestinais.

\section{Comitê de ética}

$O$ presente estudo atendeu às diretrizes e normas da Resolução no466/2012 do Conselho Nacional de Saúde do Ministério da Saúde, e foi aprovado pelo Comitê de Ética da Universidade Estadual do Maranhão sob o parecer $n$ ㅇ 1.872.625.

\section{RESULTADOS}

Foram realizados 422 exames de fezes em crianças de 0 a 11 anos de idade, sendo $187(44,3 \%)$ residentes na área de abrangência da UBS Santo Antônio, localizada na zona rural e $235(55,7 \%)$ pertencentes à área da UBS Antenor Viana, situada na zona urbana.

Das 422 crianças do estudo, a maioria pertencia à faixa etária de $4-5$ anos, 34,4\% (145), sendo que 52,8\% (223) eram do sexo feminino. Em relação à escolaridade, $44,5 \%$ (188) estavam na pré-escola, $38,0 \%$ (160) no Ensino Fundamental I e 17,5\% (74) não estudavam. 0 perfil sociodemográfico das crianças e das famílias está discriminado nas Tabelas 1 e 2.
Tabela 1 - Perfil sociodemográfico das crianças, estratificado por Unidade Básica de Saúde, Caxias-MA, 2017.

\begin{tabular}{|c|c|c|c|}
\hline \multirow{2}{*}{$\begin{array}{c}\text { Perfil } \\
\text { sociodemográfico }\end{array}$} & $\begin{array}{c}\text { UBS } \\
\text { Antenor Viana } \\
\end{array}$ & $\begin{array}{c}\text { UBS } \\
\text { Santo Antonio }\end{array}$ & Total $(n=422)$ \\
\hline & $n(\%)$ & $n(\%)$ & $n(\%)$ \\
\hline \multicolumn{4}{|l|}{ Faixa etária (anos) } \\
\hline 0 a 1 & $13(5,5)$ & $20(10,7)$ & $33(7,8)$ \\
\hline 2 a 3 & $59(25,1)$ & $28(15,0)$ & $87(20,6)$ \\
\hline 4 a 5 & $106(45,1)$ & $39(20,9)$ & $145(34,4)$ \\
\hline 6 a 7 & $22(9,4)$ & $40(21,4)$ & $62(14,7)$ \\
\hline 8 a 9 & $14(6,0)$ & $27(14,4)$ & $41(9,7)$ \\
\hline 10 a 11 & $21(8,9)$ & $33(17,6)$ & $54(12,8)$ \\
\hline \multicolumn{4}{|l|}{ Sexo } \\
\hline Feminino & $119(50,6)$ & $104(55,6)$ & $223(52,8)$ \\
\hline Masculino & $116(49,4)$ & $83(44,4)$ & $199(47,2)$ \\
\hline \multicolumn{4}{|l|}{ Escolaridade } \\
\hline Não estuda & $30(12,8)$ & $44(23,5)$ & $74(17,5)$ \\
\hline Pré-escola & $144(61,2)$ & $44(23,5)$ & $188(44,5)$ \\
\hline $\begin{array}{l}\text { Ensino } \\
\text { fundamental I }\end{array}$ & $61(26,0)$ & $99(53,0)$ & $160(38,0)$ \\
\hline
\end{tabular}

Fonte: Dados da pesquisa

Tabela 2 - Perfil sociodemográfico das famílias das crianças do estudo, estratificado por Unidade Básica de Saúde, Caxias-MA, 2017.

\begin{tabular}{|c|c|c|c|c|c|c|}
\hline \multirow[t]{2}{*}{ Variável } & \multicolumn{2}{|c|}{$\begin{array}{c}\text { UBS } \\
\text { Antenor Viana }\end{array}$} & \multicolumn{2}{|c|}{$\begin{array}{c}\text { UBS } \\
\text { Santo Antônio }\end{array}$} & \multicolumn{2}{|c|}{ Total } \\
\hline & n & $\%$ & $\mathrm{n}$ & $\%$ & $\mathbf{n}$ & $\%$ \\
\hline \multicolumn{7}{|c|}{ № de pessoas na residência } \\
\hline 2 pessoas & 7 & 2,98 & 4 & 2,14 & 11 & 2,61 \\
\hline 3 pessoas & 33 & 14,04 & 11 & 5,88 & 44 & 10,43 \\
\hline 4 pessoas & 94 & 40,00 & 35 & 18,72 & 129 & 30,57 \\
\hline 5 pessoas & 63 & 26,81 & 72 & 38,50 & 135 & 31,99 \\
\hline$<5$ pessoas & 38 & 16,17 & 65 & 34,76 & 103 & 24,41 \\
\hline \multicolumn{7}{|l|}{ Ocupação do pai } \\
\hline Aposentado & 4 & 1,70 & 14 & 7,49 & 18 & 4,27 \\
\hline Lavrador & 21 & 8,94 & 105 & 56,15 & 126 & 29,86 \\
\hline Estudante & 3 & 1,28 & 8 & 4,28 & 11 & 2,61 \\
\hline Autônomo & 50 & 21,28 & 12 & 6,42 & 62 & 14,69 \\
\hline Pedreiro & 52 & 22,13 & 15 & 8,02 & 67 & 15,88 \\
\hline Setor terciário & 19 & 8,09 & 2 & 1,07 & 21 & 4,98 \\
\hline Setor público & 5 & 2,13 & -- & -- & 5 & 1,18 \\
\hline Outras & 65 & 27,66 & 30 & 16,04 & 95 & 22,51 \\
\hline Desempregado & 16 & 6,81 & 1 & 0,53 & 17 & 4,03 \\
\hline \multicolumn{7}{|l|}{ Ocupação da mãe } \\
\hline Dona do lar & 146 & 62,13 & 144 & 77,01 & 290 & 68,72 \\
\hline Doméstica & 35 & 14,89 & 4 & 2,14 & 39 & 9,24 \\
\hline Autônoma & 2 & 0,85 & 13 & 6,95 & 15 & 3,55 \\
\hline Lavradora & 25 & 10,64 & 11 & 5,88 & 36 & 8,53 \\
\hline Setor terciário & 7 & 2,98 & -- & -- & 7 & 1,66 \\
\hline Setor pública & 6 & 2,55 & 6 & 3,21 & 12 & 2,84 \\
\hline Outras & 14 & 5,96 & 9 & 4,81 & 23 & 5,45 \\
\hline \multicolumn{7}{|l|}{ Renda familiar } \\
\hline >1 salário & 82 & 34,89 & 142 & 75,94 & 224 & 53,08 \\
\hline Até 1 salário & 130 & 55,32 & 36 & 19,25 & 166 & 39,34 \\
\hline Até 2 salários & 19 & 8,09 & 7 & 3,74 & 26 & 6,16 \\
\hline Até 3 salários & 2 & 0,85 & -- & -- & 2 & 0,47 \\
\hline Até 4 salários & 2 & 0,85 & 2 & 1,07 & 4 & 0,95 \\
\hline Total & 235 & 100,00 & 187 & 100,00 & 422 & 100,00 \\
\hline
\end{tabular}

Fonte: Dados da pesquisa

Rev. Ciênc. Méd. Biol., Salvador, v. 20, n. 2, p. 286-295, mai./ago. 2021 
A positividade entre o sexo feminino foi $51,57 \%(115)$, enquanto que no sexo masculino foi de $46,23 \%$ (92) nas
UBS Antenor Viana e Santo Antônio, porém essa diferença não teve significância estatística $(p=0,273)$ (Tabela 3).

Tabela 3 - Ocorrência de parasitos intestinais, conforme o sexo das crianças cadastradas nas áreas de abrangência das Unidades Básicas de Saúde do Antenor Viana e Santo Antônio, Caxias-MA, 2017.

\begin{tabular}{|c|c|c|c|c|c|c|c|}
\hline \multirow{3}{*}{ Variável } & \multicolumn{2}{|c|}{$\begin{array}{c}\text { UBS } \\
\text { Antenor Viana }\end{array}$} & \multicolumn{2}{|c|}{$\begin{array}{c}\text { UBS } \\
\text { Santo Antônio }\end{array}$} & \multicolumn{2}{|c|}{ Total } & \multirow{3}{*}{ P-valor } \\
\hline & Negativo & Positivo & Negativo & Positivo & Negativo & Positivo & \\
\hline & n (\%) & n (\%) & $n(\%)$ & n (\%) & n (\%) & n (\%) & \\
\hline \multicolumn{8}{|l|}{ Sexo } \\
\hline Feminino & $43(52,44)$ & $61(58,10)$ & $65(48,87)$ & $54(52,94)$ & $108(48,43)$ & $115(51,57)$ & $0,273^{a}$ \\
\hline Masculino & $39(47,56)$ & $44(41,90)$ & $68(51,13)$ & $48(47,06)$ & $107(53,77)$ & $92(46,23)$ & \\
\hline
\end{tabular}

${ }^{a}$ Teste Qui quadrado

Fonte: Dados da pesquisa

0 estrato de idade que apresentou maior quantidade de casos positivos foi a faixa etária de 4 a 5 anos, com ocorrência de $31,88 \%$ (66). Contudo, os valores das faixas etárias não diferiram estatisticamente $(p=0,770)$ (Tabela 4).

Tabela 4 - Ocorrência de parasitos intestinais, conforme faixa etária das crianças cadastradas nas áreas de abrangência das Unidades Básicas de Saúde do Antenor Viana e Santo Antônio, Caxias-MA, 2017.

\begin{tabular}{|c|c|c|c|c|c|}
\hline \multirow{2}{*}{ Resultado } & \multicolumn{2}{|c|}{ Negativo } & \multicolumn{2}{|c|}{ Positivo } & \multirow[b]{2}{*}{ P-valor } \\
\hline & $n$ & $\%$ & $n$ & $\%$ & \\
\hline \multicolumn{6}{|l|}{ Faixa etária } \\
\hline 0 a 1 ano & 16 & 7,44 & 17 & 8,21 & \\
\hline 2 a 3 anos & 46 & 21,40 & 41 & 19,81 & \\
\hline 4 a 5 anos & 79 & 36,74 & 66 & 31,88 & $0,770^{\mathrm{a}}$ \\
\hline 6 a 7 anos & 31 & 14,42 & 31 & 14,98 & \\
\hline 8 a 9 anos & 20 & 9,30 & 21 & 10,14 & \\
\hline 10 a 11 anos & 23 & 10,70 & 31 & 14,98 & \\
\hline Total & 215 & 100,00 & 207 & 100,00 & \\
\hline
\end{tabular}

${ }^{a}$ Teste Qui quadrado

Fonte: Dados da pesquisa

Os dados coletados revelaram que $49,76 \%$ (103) das crianças estavam parasitadas apenas por helmintos e $36,23 \%$ (75) somente protozoários. Em ambas as áreas de abrangência os agentes etiológicos predominantes foram os helmintos. A UBS Antenor Viana apresentou um percentual de $48,04 \%$ (49) de crianças parasitadas apenas por helmintos, enquanto a UBS Santo Antônio, essa taxa foi de 51,43\% (103) (Tabela 5).
Tabela 5 - Ocorrência de enteroparasitoses, quanto ao tipo de parasitos apresentados nas crianças cadastradas nas áreas de abrangência das Unidades Básicas de Saúde do Antenor Viana e Santo Antônio, Caxias-MA, 2017.

\begin{tabular}{lcccccc}
\hline \multirow{2}{*}{$\begin{array}{c}\text { Tipos de Parasitos } \\
\text { intestinais }\end{array}$} & \multicolumn{2}{c}{$\begin{array}{c}\text { UBS Antenor } \\
\text { Viana }\end{array}$} & \multicolumn{2}{c}{$\begin{array}{c}\text { UBS Santo } \\
\text { Antônio }\end{array}$} & \multicolumn{2}{c}{ Total } \\
\cline { 2 - 8 } & $\mathbf{n}$ & $\%$ & $\mathbf{n}$ & $\%$ & $\mathbf{n}$ & $\%$ \\
\hline Helmintos & 49 & 48,04 & 54 & 51,43 & 103 & 49,76 \\
Protozoários & 41 & 40,20 & 34 & 32,38 & 75 & 36,23 \\
$\begin{array}{l}\text { Helmintos e } \\
\text { protozoários }\end{array}$ & 12 & 11,76 & 17 & 16,19 & 29 & 14,01 \\
\hline Total & $\mathbf{1 0 2}$ & $\mathbf{1 0 0 , 0 0}$ & $\mathbf{1 0 5}$ & $\mathbf{1 0 0 , 0 0}$ & $\mathbf{2 0 7}$ & $\mathbf{1 0 0 , 0 0}$ \\
\hline
\end{tabular}

Fonte: Dados da pesquisa

Dentre os helmintos, o parasito mais frequente foi $A$. lumbricoides com taxa $68,18 \%$ (90) de positividade. Além disso, o A. lumbricoides também ocorreu em associação com outros helmintos. Quanto aos protozoários, a maior ocorrência foi de Entamoeba sp., 65,38\% (68). Observou-se que em ambas as áreas ocorreram as mesmas espécies de parasitos, embora com frequências diferentes. Tanto na área rural como na urbana o helminto prevalente foi o A. lumbricoides, com taxa de positividade de $63,38 \%$ (45) e $73,77 \%$ (45), respectivamente. Quanto aos protozoários, o mais frequente nas duas UBS foi a Entamoeba sp., sendo encontrado em $60,78 \%$ (31) das amostras da UBS Santo Antônio e, em 69,81\% (37) dos casos da UBS Antenor Viana (Tabela 6). 
Tabela 6 - Helmintos e protozoários gastrintestinais em crianças de 0 a 11 anos, estratificados por áreas de abrangências das Unidades Básicas de Saúde do Antenor Viana e Santo Antônio, Caxias-MA, 2017.

\begin{tabular}{|c|c|c|c|c|c|c|}
\hline \multirow[t]{2}{*}{ Agentes etiológicos } & \multicolumn{2}{|c|}{$\begin{array}{c}\text { UBS } \\
\text { Antenor Viana }\end{array}$} & \multicolumn{2}{|c|}{$\begin{array}{c}\text { UBS } \\
\text { Santo Antônio }\end{array}$} & \multicolumn{2}{|c|}{ Total } \\
\hline & n & $\%$ & n & $\%$ & n & $\%$ \\
\hline \multicolumn{7}{|l|}{ Helminto } \\
\hline Ascaris lumbricoides & 45 & 73,77 & 45 & 63,38 & 90 & 68,18 \\
\hline Enterobius vermiculares & 5 & 8,20 & 7 & 9,86 & 12 & 9,09 \\
\hline Ancilostomídeos & 4 & 6,56 & 6 & 8,45 & 10 & 7,75 \\
\hline Trichurus trichiura & 2 & 3,28 & 5 & 7,04 & 7 & 5,30 \\
\hline Hyminolepis nana & 2 & 3,28 & 4 & 5,63 & 6 & 4,55 \\
\hline Strongyloides stercoralis & 1 & 1,64 & 2 & 2,82 & 3 & 2,27 \\
\hline Ascarislumbricoides/Enterobius vermiculares & 2 & 3,28 & 1 & 1,41 & 3 & 2,77 \\
\hline Ascaris lumbricoides/Hyminolepis nana & - & - & 1 & 1,41 & 1 & 0,76 \\
\hline Total & 61 & 100,00 & 71 & 100,00 & 132 & 100,00 \\
\hline \multicolumn{7}{|l|}{ Protozoário } \\
\hline Entamoeba sp. & 37 & 69,81 & 31 & 60,78 & 68 & 65,38 \\
\hline Giardia lamblia & 11 & 20,75 & 11 & 21,57 & 22 & 21,15 \\
\hline Endolimax nana & 5 & 9,43 & 6 & 11,76 & 11 & 10,58 \\
\hline Giardia lamblia/Entamoeba sp. & - & - & 2 & 3,92 & 2 & 1,92 \\
\hline Entamoeba sp./Endolimax nana & - & - & 1 & 1,96 & 1 & 0,96 \\
\hline Total & 53 & 100,00 & 51 & 100,00 & 104 & 100,00 \\
\hline
\end{tabular}

Fonte: Dados da pesquisa

Os dados relativos a associação entre a ocorrência crianças estão discriminados na Tabela 7. de parasitos intestinais e o perfil sociodemográfico das

Tabela 7 - Associação entre a ocorrência de parasitoses e as características sociodemográficas das crianças cadastradas nas áreas de abrangência das Unidades Básicas de Saúde do Antenor Viana e Santo Antônio, Caxias-MA, 2017.

\begin{tabular}{|c|c|c|c|c|}
\hline \multirow[b]{2}{*}{ Características sociodemográficas das crianças } & \multicolumn{2}{|c|}{ Resultado do exame } & \multirow[b]{2}{*}{ p-valor* } & \multirow[b]{2}{*}{ OR (IC 95\%) } \\
\hline & $\begin{array}{c}\text { Positivo } \\
\text { n (\%) }\end{array}$ & $\begin{array}{c}\text { Negativo } \\
\text { n (\%) }\end{array}$ & & \\
\hline \multicolumn{5}{|l|}{ UBS } \\
\hline Antenor Viana & $102(49,3)$ & $133(61,9)$ & - & 1 \\
\hline Santo Antônio & $105(50,7)$ & $82(38,1)$ & 0,009 & $1,670(1,134-2,459)$ \\
\hline \multicolumn{5}{|l|}{ Faixa etária (anos) } \\
\hline 0 a 1 & $17(8,2)$ & $16(7,4)$ & - & 1 \\
\hline 2 a 3 & $41(19,8)$ & $46(21,4)$ & 0,668 & $0,839(0,376-1,871)$ \\
\hline 4 a 5 & $66(31,9)$ & $79(36,8)$ & 0,534 & $0,786(0,369-1,676)$ \\
\hline 6 a 7 & $31(15,0)$ & $31(14,4)$ & 0,888 & $0,941(0,404-2,191)$ \\
\hline 8 a 9 & $21(10,1)$ & $20(9,3)$ & 0,980 & $0,988(0,395-2,473)$ \\
\hline 10 a 11 & $31(15,0)$ & $23(10,7)$ & 0,592 & $1,269(0,531-3,028)$ \\
\hline \multicolumn{5}{|l|}{ Sexo } \\
\hline Feminino & $115(55,6)$ & $108(50,2)$ & 0,273 & $1,238(0,844-1,816)$ \\
\hline Masculino & $92(44,4)$ & $107(49,8)$ & - & 1 \\
\hline \multicolumn{5}{|l|}{ Escolaridade } \\
\hline Não estuda & $40(19,3)$ & $34(15,8)$ & - & 1 \\
\hline Pré-escola & $83(40,1)$ & $105(48,8)$ & 0,149 & $0,672(0,391-1,153)$ \\
\hline Ensino fundamental & $84(40,6)$ & $76(35,3)$ & 0,825 & $0,939(0,541-1,632)$ \\
\hline \multicolumn{5}{|l|}{ Renda familiar (salários mínimos) } \\
\hline$<1$ & $119(57,5)$ & $105(48,9)$ & 0,116 & $5,667(0,652-49,285)$ \\
\hline 1 & $77(37,2)$ & $89(41,4)$ & 0,186 & $4,326(0,495-37,834)$ \\
\hline 2 & $10(4,8)$ & $16(7,4)$ & 0,329 & $3,125(0,317-30,791)$ \\
\hline$>3$ & $1(0,5)$ & $5(2,3)$ & - & 1 \\
\hline
\end{tabular}

*Teste Qui-Quadrado. Valores estatisticamente significativos $(p<0,05)$ em negrito. OR-Odds ratio; IC - Intervalo de Confiança

Fonte: Dados da pesquisa 
Na análise da associação entre a ocorrência de parasitos intestinais e as condições de saneamento básico e a higiene das crianças, observou-se que o destino do lixo, abastecimento de água, tratamento de água no domicílio, local das instalações sanitárias e pavimentação das ruas, apresentaram significância estatística para a ocorrência de parasitoses (Tabela 8).

Neste estudo, 53,1\% (110) dos casos positivos ocorreram em crianças cujo destino do lixo na sua residência era a incineração $(p<0,001)$, sendo que a maioria, $50,7 \%$ (105) residiam em lares em que o abastecimento de água era proveniente de poço artesiano $(p=0,009)$. Quando analisado o tipo de tratamento de água no domicilio, observou-se maior positividade em crianças que ingeriam água sem nenhum tratamento, $67,1 \%(139)(p<0,001)$. Dentre os indivíduos que possuíam algum tipo instalação sanitária, observou-se que $56,8 \%$ (88) dos casos ocorreu em indivíduos onde estas se localizavam fora da residência $(p=0,049)$. Das 207 crianças que apresentaram algum enteroparasito, $77,3 \%(160)$ residiam em locais que não possuíam pavimentação nas ruas $(p<0,001)$ (Tabela 8$)$.

Tabela 8 - Associação entre a ocorrência de parasitoses e as condições de saneamento básico e higiene das crianças cadastradas nas áreas de abrangência das Unidades Básicas de Saúde do Antenor Viana e Santo Antônio, Caxias-MA, 2017.

\begin{tabular}{|c|c|c|c|c|}
\hline \multirow{3}{*}{$\begin{array}{l}\text { Condições de saneamento } \\
\text { básico e higiene }\end{array}$} & \multicolumn{2}{|c|}{ Resultado do exame } & \multirow{3}{*}{ p-valor* } & \multirow{3}{*}{ OR (IC 95\%) } \\
\hline & $\begin{array}{l}\text { Positivo } \\
(n=207)\end{array}$ & $\begin{array}{l}\text { Negativo } \\
(n=215)\end{array}$ & & \\
\hline & $\mathrm{n}(\%)$ & $\mathrm{n}(\%)$ & & \\
\hline \multicolumn{5}{|l|}{ Destino do lixo } \\
\hline Coleta pública & $78(37,7)$ & $120(55,8)$ & - & 1 \\
\hline Incinerado & $110(53,1)$ & $77(35,8)$ & $<0,001$ & $2,198(1,462-3,303)$ \\
\hline Enterrado & $1(0,5)$ & $4(1,9)$ & 0,397 & $0,385(0,042-3,505)$ \\
\hline Céu aberto & $18(8,7)$ & $14(6,5)$ & 0,076 & $1,978(0,930-4,206)$ \\
\hline \multicolumn{5}{|l|}{ Abastecimento de água } \\
\hline Poço artesiano & $105(50,7)$ & $82(38,1)$ & 0,009 & $1,670(1,134-2,459)$ \\
\hline Rede pública & $102(49,3)$ & $133(61,9)$ & - & 1 \\
\hline \multicolumn{5}{|l|}{ Tratamento de água no domicílio } \\
\hline Filtrada & $43(20,8)$ & $110(51,2)$ & - & 1 \\
\hline Clorada & $2(1,0)$ & $1(0,5)$ & 0,187 & $5,116(0,452-57,895)$ \\
\hline Coada & $23(11,1)$ & $19(8,8)$ & 0,002 & $3,097(1,534-6,251)$ \\
\hline Sem tratamento & $139(67,1)$ & $85(39,5)$ & $<0,001$ & $4,183(2,684-6,521)$ \\
\hline \multicolumn{5}{|l|}{ Destino das fezes e da urina } \\
\hline Fossa & $144(69,6)$ & $150(69,8)$ & - & 1 \\
\hline Céu aberto & $52(25,1)$ & $52(24,2)$ & 0,858 & $1,042(0,666-1,629)$ \\
\hline Outros & $11(5,3)$ & $13(6,0)$ & 0,767 & $0,881(0,382-2,031)$ \\
\hline \multicolumn{5}{|l|}{ Local das instalações sanitárias(n=319) } \\
\hline Dentro da residência & $67(43,2)$ & $89(54,3)$ & - & 1 \\
\hline Fora da residência & $88(56,8)$ & $75(45,7)$ & 0,049 & $1,559(1,002-2,425)$ \\
\hline \multicolumn{5}{|l|}{ Pavimentação das ruas } \\
\hline Sim & $47(22,7)$ & $90(41,9)$ & - & 1 \\
\hline Não & $160(77,3)$ & $125(58,1)$ & $<0,001$ & $2,451(1,605-3,743)$ \\
\hline \multicolumn{5}{|l|}{ Anda descalço } \\
\hline Sim & $171(82,6)$ & $146(67,9)$ & 0,001 & $2,245(1,418-3,554)$ \\
\hline Não & $36(17,4)$ & $69(32,1)$ & - & 1 \\
\hline \multicolumn{5}{|l|}{ Lava as mãos após o uso do banheiro } \\
\hline Não & $26(12,6)$ & $9(4,2)$ & 0,003 & $3,288(1,501-7,200)$ \\
\hline Quando solicitado & $181(87,4)$ & $206(95,8)$ & - & 1 \\
\hline \multicolumn{5}{|l|}{ Lava as mãos antes das refeições } \\
\hline Sim & $62(30,0)$ & $150(69,8)$ & - & 1 \\
\hline Não & $46(22,2)$ & $14(6,5)$ & $<0,001$ & $7,949(4,078-15,495)$ \\
\hline Quando solicitado & $99(47,8)$ & $51(23,7)$ & $<0,001$ & 4,696 (2,997-7,359) \\
\hline
\end{tabular}

*Teste Qui-Quadrado. Valores estatisticamente significativos $(p<0,05)$ em negrito; OR - Odds ratio; IC - Intervalo de Confiança.

Fonte: Dados da pesquisa 
Verificou-se maior número de casos de enteroparasitoses, 82,6\% (171), em crianças que andavam sem calçados $(p<0,001)$. Em relação ao costume de lavar as mãos após o uso do banheiro, observou-se que $87,4 \%$ (181) dos indivíduos parasitados não as higienizavam em todas as ocasiões, sendo que as demais, 12,6\% (26), não possuíam esse hábito $(p<0,003)$. Do mesmo modo, notou-se que a maioria das crianças com parasito intestinal não lavavam as mãos adequadamente antes das refeições, uma vez que, $47,8 \%$ (99) destas somente as higienizavam quando era solicitado $(\mathrm{p}<$ 0,001) (Tabela 8).

Os resultados da análise multivariada por regressão logística estão expostos na Tabela 9, a qual mostra as variáveis que permaneceram no modelo após o ajuste realizado. Conforme observa-se, somente as variáveis ingestão de água sem tratamento no domicilio ( $\mathrm{OR}=3,749$; $p<0,001)$ e a não lavagem das mãos antes das refeições $(O R=4,670 ; p=<0,001)$ se mostraram fatores de risco para o desenvolvimento de parasitoses intestinais.

Tabela 9-Regressão logística entre variáveis socioeconômicas, saneamento básico e aspectos de higiene das crianças cadastradas nas áreas de abrangência das Unidades Básica de Saúde do Antenor Viana e Santo Antônio, Caxias-MA, 2017.

\begin{tabular}{|c|c|c|c|c|}
\hline \multirow{2}{*}{ Variáveis } & \multicolumn{2}{|c|}{ Análise não ajustada } & \multicolumn{2}{|c|}{ Análise ajustada } \\
\hline & OR (IC 95\%) & p-valor* & OR (IC 95\%) & p-valor* \\
\hline \multicolumn{5}{|c|}{ Tratamento de água no domicílio } \\
\hline Filtrada & 1 & - & 1 & - \\
\hline Clorada & $5,116(0,452-57,895)$ & 0,187 & $2,060(0,467-9,090)$ & 0,340 \\
\hline Coada & $3,097(1,534-6,251)$ & 0,002 & $4,554(0,181-114,764)$ & 0,357 \\
\hline Sem tratamento & $4,183(2,684-6,521)$ & $<0,001$ & $3,749(1,819-7,727)$ & $<0,001$ \\
\hline \multicolumn{5}{|c|}{ Lava as mãos antes das refeições } \\
\hline Sim & 1 & - & 1 & - \\
\hline Não & $7,949(4,078-15,495)$ & $<0,001$ & $4,670(2,176-10,022)$ & $<0,001$ \\
\hline Às vezes & $4,696(2,997-7,359)$ & $<0,001$ & $1,034(0,354-3,021$ & 0,952 \\
\hline
\end{tabular}

*Teste Qui-Quadrado. Valores estatisticamente significativos $(p<0,05)$ em negrito; OR - Odds ratio; IC - Intervalo de Confiança.

\section{DISCUSSÃO}

A frequência de parasitoses intestinais encontrada nesta investigação é considerada alta, no entanto, se mostra similar aos resultados de outros estudos epidemiológicos realizados em diferentes regiões do Brasil ${ }^{11-13}$. No entanto, percentuais inferiores são registrados na literatura ${ }^{3-5,14,15}$.

Quanto à ocorrência de casos positivos por áreas de abrangências estudadas, observou-se que na zona rural a frequência de casos positivos, foi maior em relação à área urbana. Entretanto, a análise de regressão logística mostrou que não houve associação significativa entre os casos positivos nas áreas rurais. Resultados diferentes foram observados em estudo com crianças e adolescentes do ensino fundamental no município de São João del-Rei-MG, onde uma diferença significante entre as prevalências nas áreas rurais, $36,4 \%$, e urbana, $23,5 \%{ }^{16}$ foi observada.

As diferenças entre a prevalência de parasitoses intestinais entre as áreas urbanas e rurais, possivelmente, refletem o menor nível socioeconômico, as condições mais inapropriadas de higiene e saneamento básico na zona rural, os quais podem favorecer a infecção por enteroparasitos. Dessa forma, nas populações rurais as enteroparasitoses são mais acentuadas, devido ao acesso precário à informação, aos serviços de saúde e ao saneamento básico ${ }^{17}$. Por outro lado, uma vez que existam condições socioeconômicas, ambientais e educacionais adequadas, a prevalência em áreas rurais poderá ser similar ou até mesmo inferior a áreas urbanizadas $^{16}$.
Neste estudo, a maioria das crianças estava parasitada apenas por helmintos, fato este também observado por outros autores ${ }^{18,19}$. Pode se inferir, que as taxas elevadas de helmintos possivelmente estejam associadas às precárias condições sanitárias das áreas onde ocorreu o estudo. Por outro lado, estudos mais recentes apontam maior ocorrência de infecção por protozoários $3-5,14,15,20,21$. Há registro na literatura de infecção somente por protozoários ${ }^{22}$

Dentre os parasitos encontrados, neste estudo, o helminto $A$. lumbricoides foi o mais frequente $(68,18 \%)$. Esse alto percentual registrado para este agente etiológico reflete diretamente na capacidade de propagação dessa espécie, a qual ocorre principalmente através da ingestão de água e alimentos contaminados com os ovos viáveis do parasito, aliado à resistência dos ovos às condições ambientais, permanecendo por longos períodos viáveis no ambiente possibilitando assim a infecção dos hospedeiros.

O helminto $A$. lumbricoides causa a doença denominada ascaridíase ${ }^{23}$. Na maioria dos casos essa helmintíase é leve e clinicamente benigna, entretanto pode ocorrer complicações devido a lesões de natureza obstrutiva ${ }^{24}$. Quando sintomática, o sinal mais comum é dor abdominal vaga. Além disso, esses helmintos podem causar alterações inflamatórias na mucosa intestinal, ocasionando alteração na secreção e na motilidade do intestino, resultando em diarreia, má absorção e perda de apetite. A gravidade dos sintomas relaciona-se diretamente ao número de parasitos adultos que o indivíduo alberga. Infecções maciças por muitos vermes podem resultar em 
bloqueio mecânico do intestino delgado especialmente em crianças $^{25}$.

Estudos afirmam que o A. lumbricoides é o helminto mais prevalente de todos os enteroparasitos que acometem o homem em países com baixas condições socioeconômicas ${ }^{26}$. Em virtude dessa alta prevalência, assim como das complicações referentes à ascaridíase, é imprescindível alertar a população quanto as medidas de prevenção dessa doença.

Em referência aos protozoários, a maior ocorrência foi de Entamoeba sp., com $65,38 \%$ de casos positivos nas amostras pesquisadas. Cabe ressaltar que, no presente estudo não se identificou as espécies de amebas, ficando a identificação em nível de gênero. Entretanto, estudos epidemiológicos realizados por outros pesquisadores apontam para uma maior prevalência da Entamoeba coli em relação ao complexo Entamoeba histolytica/Entamoeba dispar ${ }^{19,27} 28$.

Embora várias espécies de amebas possam habitar o intestino humano, apenas a $E$. histolytica é patogênica para o homem ${ }^{29}$. Esse protozoário causa amebíase, doença que pode ser acompanhada ou não de manifestações clinícas ${ }^{25} .0$ quadro sintomático varia de uma forma branda, caracterizada por desconforto abdominal leve ou moderado, com sangue e/ou muco nas dejeções, até uma diarreia aguda e fulminante, de caráter sanguinolento ou mucoide, acompanhada de febre e calafrios. Nos casos graves, as formas trofozoíticas se disseminam pela corrente sanguínea, provocando abscesso no fígado, nos pulmões ou cérebro. Quando não diagnosticadas e tratadas, podem levar o paciente a óbito ${ }^{30}$.

Quanto a $E$. coli, embora esta seja uma espécie comensal que habita o intestino do homem, a sua elevada frequência pode indicar aspectos inadequados de higiene, bem como ao consumo de água e alimentos contaminados com os cistos deste parasito, uma vez que a principal forma de transmissão destes agentes ocorre pela via fecal-oral ${ }^{31,32}$. Dessa forma, essa espécie pode ser considerada um importante indicador das condições socioeconômicas, ambientais e sanitárias de uma população ${ }^{33}$. Além disso, parasitos patogênicos que tem a mesma forma de transmissão fecal-oral que os comensais podem ser disseminados na população ${ }^{14}$.

Em referência ao parasitismo por Giardia lamblia os percentuais de ocorrência são variáveis, conforme os dados disponíveis na literatura ${ }^{4,5,15,20-22}$. É valido ressaltar que, o diagnóstico de giardíase quando realizado com uma única amostra comumente apresenta baixos índices de positividade, uma vez que os indivíduos parasitados não eliminam cistos continuamente ${ }^{26}$. Contudo, no Brasil, a prevalência $G$. lamblia pode variar de acordo com a população e a região, de maneira geral, situa-se entre 9 e $50 \%{ }^{34}$.

As infeções causadas pela G. lamblia ocorrem com maior frequência em faixas etárias inferiores a 10 anos, sendo rara durante os primeiros anos de idade devido ao aleitamento materno ${ }^{29}$. Ainda que a giardíase seja normalmente assintomática, causando diarreia aquosa que regride espontaneamente ${ }^{24}$, é necessário diagnosticar e tratar adequadamente os pacientes infectados, uma vez que, a persistência de muitos parasitos no intestino pode levar a avitaminose, pela diminuição da superfície de absorção de lipídeos e vitaminas lipossolúveis ${ }^{23}$.

Nas áreas de abrangência pesquisadas, notou-se que a maioria das crianças apresentaram monoparasitismo. Este achado é comum em grande parte dos estudos sobre enteroparasitos ${ }^{4,16,35}$, que demostraram ocorrência de apenas uma espécie de parasito intestinal.

Destaca-se que, a ocorrência de casos de biparasitismo e poliparasitismo é um dado epidemiológico que indica o agravamento das condições de saúde da população exposta a essas infecções. $O$ hospedeiro humano pode albergar diferentes espécies de parasitos intestinais, sendo que, quanto mais contaminado o ambiente externo maior a probabilidade de infecções com poliparasitismo ${ }^{36}$.

$\mathrm{Na}$ análise hierarquizada neste estudo, permaneceram como fatores de risco para a ocorrência de parasitoses intestinais a ingestão de água sem tratamento no domicílio e a não lavagem das mãos antes das refeições. Em relação à ingestão de água, observou-se que as crianças que ingeriam água sem tratamento domiciliar apresentavam chance 3,7 vezes maior para adquirir parasitoses que as crianças que tomavam água tratada filtrada. $O$ uso do filtro de água nas residências mostra-se fortemente associado à redução da prevalência de helmintos e protozoários ${ }^{16}$. Os indivíduos que ingerem água não filtrada apresentavam a possibilidade 15,9 vezes maior de adquirir parasitoses ${ }^{37}$. Existe associação significante entre o consumo de água da torneira e a presença de infecções por enteroparasi$\operatorname{tos}^{38}$. Portanto, a qualidade da água usada no consumo doméstico é essencial para prevenção e diminuição da incidência das parasitoses, uma vez que a maioria destas tem a água como principal veículo de transmissão.

Quanto a não lavagem das mãos antes das refeições, neste estudo observou-se chance de ocorrência 4,7 vezes maior para o desenvolvimento de parasitoses intestinais em relação às crianças que possuíam o hábito de lavar as mãos. Alta frequência de enteroparasitos em crianças de 2 a 6 anos, com chance 1,5 vezes maior para crianças que não lavavam as mãos antes das refeições e após o uso do sanitário já foi observada em estudos sobre enteroparasitoses infantis ${ }^{39}$. Da mesma forma, pesquisa com crianças de áreas urbana e rural da rede municipal de Rolândia-PR, apontou o hábito de lavar as mãos antes das refeições como um fator protetor contra enteroparasitoses em áreas rurais ${ }^{40}$.

Os estudos reforçam a importância da lavagem das mãos para a prevenção destas doenças, considerando o encontro de formas imaturas de parasitos gastrintestinais em material subungeal de crianças ${ }^{41,42}$. Esses dados reforçam a necessidade da adoção de hábitos adequados de higiene, como a lavagem das mãos e corte das unhas, como medidas eficazes para a prevenção das enteroparasitoses. 
Estudos recentes sobre fatores de risco associados a enteropatias parasitárias, também apontam a importância da qualidade da água para consumo da população e as práticas de higiene, especialmente o hábito de lavar as mãos antes das refeições e após o uso do banheiro ${ }^{3,4,5,21}$. Fatores como infraestrutura de saúde ${ }^{3}$, renda familiar, faixa etária, hábito das crianças de frequentar córregos ${ }^{14}$ e ausência de saneamento básico ${ }^{21}$ também são apontados como condicionantes de risco para a ocorrência de parasitoses intestinais. Outros fatores também podem está associados à presença de parasitos intestinais, como a baixa escolaridade da mãe, a presença de animais domésticos em casa, o tempo de gestação inferior a 36 semanas e o tempo de amamentação ${ }^{22}$.

\section{CONCLUSÃO}

A ocorrência de parasitoses intestinais foi considerada elevada, com maior frequência na zona rural. Diante dos resultados, constatou-se que as áreas em estudo são altamente vulneráveis para a ocorrência de infecção por enteroparasitos, principalmente em decorrência dos aspectos socioeconômico, das condições insatisfatórias de saneamento e dos hábitos inadequados de higiene apresentados pela maioria das crianças.

Nesta pesquisa verificou-se que a ingestão de água sem tratamento no domicílio e a não lavagem das mãos antes das refeições foram os prováveis fatores de risco associados à alta ocorrência das infecções parasitárias nestes locais.

O estudo adverte sobre necessidade de implementação de políticas públicas, em virtude dos elevados índices de enteroparasitoses nestas crianças. Essas medidas de controle devem enfatizar a importância dos hábitos adequados de higiene e a melhoria do saneamento básico desses locais. Para tanto, é importante o engajamento de todos, como os órgãos governamentais, educadores, profissionais da saúde e familiares, buscando reduzir ou eliminar a prevalência das infecções parasitárias na população.

\section{AGRADECIMENTOS}

Á Coordenação de Aperfeiçoamento de Pessoal de Nível Superior/CAPES e a Fundação de Apoio ao Desenvolvimento Científico e tecnológico do Maranhão/FAPEMA.

\section{REFERÊNCIAS}

1. ABREU, L. K. et al. Prevalência e aspectos sócio-epidemiológicos de enteroparasitoses em crianças do centro municipal de educação infantil em Janiópolis - PR. SaBios: Rev. Saúde e Biol., Campos Mourão, v. 9, n. 3, p. $76-84$, out./dez. 2014.

2. BRASIL. Ministério da Saúde. Departamento de Informática do SUS (DATASUS). Mortalidade Infantil. Brasília: Ministério da Saúde, 2016. Disponível em: http://tabnet.datasus.gov.br/cgi/tabcgi.exe?sim/cnv/ inf10uf.def. Acesso em: 12 set . 2017.

3. BANHOS, E. F. et al. Prevalência e fatores de risco para infecções por parasito intestinal em crianças de escolas na cidade de Santarém, estado do Pará, Brasil. ABCS Health Sci., Santo André, v. 42, n. 3, p. 137-142, dez. 2017.

4. DELAZERI, M. A. F.; LAWISCH, G. K. S. Incidência de parasitos intestinais em crianças das escolas municipais de educação infantil e ensino fundamental de um município do interior do Rio Grande do Sul. Destaques Acadêmicos, Lajeado, v. 9, n. 3, p. 206-215, 2017.

5. BARBOSA, J. A. et al. Análise do perfil socioeconômico e da prevalência de enteroparasitoses em crianças com idade escolar em um município de Minas Gerais. HU Rev., Juiz de Fora, v. 43 n .3, p. 391-397, set. 2017.

6. BRASIL. Ministério da Saúde. Departamento de Atenção Básica. Política Nacional de Atenção Básica. Brasília: Ministério da Saúde, 2012.

7. HEUKELBACH, J.; OLIVEIRA, F.; FELDMEIER, H. Ectoparasitoses e saúde pública no Brasil: desafios para controle. Cad. Saúde Pública, Rio de Janeiro, v. 19, n. 5, p. 1535-1540, out. 2003.

8. BRASIL. Ministério da Saúde. Departamento de Atenção Básica. Índice de Desenvolvimento Humano Municipal Brasileiro: série atlas do desenvolvimento humano no Brasil 2013. Brasília: PNUD, Ipea, 2013. 96 p. Disponível em: http://atlasbrasil.org.br/2013/. Acesso em: 24 out. 2017.

9. SILVA, F. S. et al. Frequência de parasitos intestinais no município de Chapadinha, Maranhão, Brasil. Rev. Patol. Trop., Goiania, v. 39, n. 1, p. 63-68, jan./mar. 2010.

10. HOFFMAN, W. A.; PONS, J. A.; JANER, J. L. The sedimentationconcentration method in schistosomiasis mansoni. J. Pub. Health., Porto Rico, v. 9, n. 3, p. 283-289, 1934.

11. CARVALHO, T. B.; CARVALHO, L. R.; MASCARINI, L. M. Occurrence of enteroparasites in day care centers in Botucatu (São Paulo, State, Brazil) with emphasis on cryptosporidium sp., giardia duodenalis and enterobius vermicularis. Rev. Inst. Med. Trop. São Paulo, São Paulo, v. 48 , n. 5, p. 269-273, set./out. 2006.

12. SILVA, R. R. et al. Prevalência de parasitoses e estado nutricional de pré-escolares de centros educacionais municipais no sul de Minas Gerais. Nutrire Rev. Soc. Bras. Aliment. Nutr., São Paulo, v. 35, n. 1, p. 59-72, abr. 2010.

13. UCHÔA, C. M. A. et al. Parasitoses intestinais: prevalência em creches comunitárias da cidade de Niterói, Rio de Janeiro - Brasil. Rev. Inst. Adolfo Lutz, São Paulo, v. 60, n. 2, p. 97-101, 2001.

14. LOPES-MORI, F. M. R. et al. Fatores associados a enteroparasitoses em escolares da rede municipal de ensino de Cambé. Semina Cienc. Biol. Saúde, Londrina, v. 37, n. 1, p.15-24, jan./jun. 2016.

15. PIRES, E. da C. R. et al. Abordagem interdisciplinar das parasitoses intestinais em escolares da microrregião de Sete Lagoas-MG. Arq. Ciênc. Saúde UNIPAR, Umuarama, v. 20, n. 2, p. 111-116, maio/ago. 2016.

16. BELO, V. S. et al. Fatores associados à ocorrência de parasitoses intestinais em uma população de crianças e adolescentes. Rev. Paul. Pediatr., São Paulo, v. 30, n. 2, p. 195-201, jun. 2012.

17. OLIVEIRA, S. H. S. D. et al. Complicação de abscesso hepático em criança ribeirinha poliparasitada: um relato de caso e discussão sobre as condições de saneamento e acesso à saúde dessa população. Rev. Bras. Med. Fam Comunidade, Rio de Janeiro, v. 9, n. 31, p. 213-218, abr./jun. 2014.

18. BASSO, R. M. C. et al. Evolução da prevalência de parasitoses intestinais em escolares em Caxias do Sul, RS. Rev. Soc. Bras. Med. Trop., Brasília, v. 41, n. 3, p. 263-268, jun. 2008.

19. SANTOS, F. S. et al. Prevalência de enteroparasitismo em crianças de comunidades ribeirinhas do Município de Coari, no médio Solimões, Amazonas, Brasil. Rev. Pan-Amazônica Saúde, Levilândia, v. 1, n. 4, p. 23-28, dez. 2010. 
20. CAVAGNOLLI, N. I. et al. Prevalência de enteroparasitoses e análise socioeconômica de escolares em Flores da Cunha-RS. Rev. Patol. Trop., Goiania, v. 44, n. 3, p. 312-322, out. 2015.

21. CROZARA, R. S. S. et al. Ocorrência de enteroparasitoses em crianças atendidas pelo programa de erradicação do trabalho infantil (peti) nos distritos de Interlândia e Sousânia na cidade de Anápolis-GO no ano de 2013. Enciclopédia Biosfera, Goiânia, v. 13, n. 23, p. 888-897, 2016.

22. FONSECA, T. C. et al. Fatores associados às enteroparasitoses em crianças usuárias de creches comunitárias. Ciência \& Saúde, Porto Alegre, v. 11, n. 1, p. 33-40, jan./mar. 2018.

23. NEVES, D. P. et al. Parasitologia humana. 11. ed. São Paulo: Atheneu, 2005. $493 \mathrm{p}$

24. REY, L. Bases da parasitologia médica. 3. ed. Rio de Janeiro: Guanabara Koogan, 2010. 391 p.

25. FERREIRA, M. U.; FORANDA, A. S.; SHUMAKER, T. T. S. Fundamentos biológicos da parasitologia humana. São Paulo: Manole, 2003. 155 p.

26. CARRILLO, M. R. G. G.; LIMA, A. A.; NICOLATO, R. L. C. Prevalência de enteroparasitoses em escolares do bairro Morro de Santana no Município de Ouro Preto, MG. Rev. Bras. Anal. Clin., Rio de Janeiro, v. 37, n. 3, p. 191-193, 2005.

27. FURTAdo, L. F. V.; MelO, A. C. F. L. Prevalência e aspectos epidemiológicos de enteroparasitoses na população geronte de Parnaíba, Estado do Piauí. Rev. Soc. Bras. Med. Trop., Brasília, v. 44, n. 4, p. 513-515, jul./ago. 2011.

28. VISSER, S.; GIATTI, L. L. CARVALHO, R. A. C.; GUERREIRO, J. C. H. Estudo da associação entre fatores socioambientais e prevalência de parasitose intestinal em área periférica da cidade de Manaus (AM, Brasil). Ciênc. Saúde Colet., Rio de Janeiro, v. 16, n. 8, p. 3481-3492, ago. 2011.

29. CIMERMAN, S.; CIMERMAN, B. Medicina tropical. São Paulo: Atheneu, 2003. 699 p.

30. BRASIL. Ministério da Saúde. Secretaria de Vigilância em Saúde. Departamento de Vigilância Epidemiológica. Doenças infecciosas e parasitárias: guia de bolso. 8. ed. Brasília: Ministério da Saúde, 2010. $444 \mathrm{p}$.

31. MACEDO, H. S. Prevalência de parasitos e comensais intestinais em crianças de escolas da rede pública municipal de Paracatu (MG). Rev.
Bras. Anál. Clín., Rio de Janeiro, v. 37, n. 4, p. 209-213, out./dez.2005.

32. VASCONCELOS, I. A. B. et al. Prevalência de parasitoses intestinais entre crianças de 4-12 anos no Crato, Estado do Ceará: um problema recorrente de saúde pública. Acta Sci., Health Sci., Maringá, v. 33, n. 1, p. 35-41, 2011.

33. PEDRAZA, D. F.; QUEIROZ, D.; SALES, M. C. Doenças infecciosas em crianças pré-escolares brasileiras assistidas em creches. Ciênc. Saúde Colet., Rio de Janeiro, v. 19, n. 2, p. 511-528, fev. 2014.

34. PITTNER, E. et al. Enteroparasitoses em crianças de uma comunidade escolar na cidade de Guarapuava, PR. Rev. Salus, Guarapuava, v. 1, n. 1, p. 97-100, jan./jun. 2007.

35. SILVA, F. M. A. et al. Aspectos epidemiológicos e prevalência de enteroparasitoses em crianças do Bairro Jambeiro, São Luís, MA. Rev. Ciênc. Saúde, São Luís, v. 13, n. 2, p. 123-130, jul./dez. 2011.

36. SANTOS, A. S.; MERLINI, L. S. Prevalência de enteroparasitoses na população do município de Maria Helena, Paraná. Ciênc. Saúde Colet., Rio de Janeiro, v. 15, n. 3, p. 899-905, jan./maio 2010.

37. KOMAGOME, S. H. et al. Fatores de risco para infecção parasitária intestinal em crianças e funcionários de creche. Ciênc. Cuid. Saúde, Maringá, v. 6, supl. 2, p. 442-447, 2007.

38. BELLOTO, M. V. T. et al. Enteroparasitoses numa população de escolares da rede pública de ensino do Município de Mirassol, São Paulo, Brasil. Rev. Pan-Amazônica Saúde, Levilândia, v. 2, n. 1, p. 3744, mar. 2011.

39. GURGEL, R. Q. et al. Creche: ambiente expositor ou protetor nas infestações por parasitos intestinais em Aracaju, SE. Rev. Soc. Bras. Med. Trop., Brasília, v. 38, n. 3, p. 267-269, maio 2005.

40. GIRALDI, N. et al. Enteroparasites prevalence among daycare and elementary school children of municipal schools, Rolândia, PR, Brazil. Rev. Soc. Bras. Med. Trop., Brasília, v. 34, n. 4, p. 385-387, jul./ago. 2001.

41. BEZERRA, F. S. M. et al. Incidência de parasitos intestinais em material sub-ungueal e fecal em crianças da Creche Aprisco - Fortaleza, CE. Rev. Bras. Anal. Clin., Rio de Janeiro, v. 35, n. 1, p. 39-40, 2003.

42. SILVA-SOUZA, N. et al. Ocorrência de enteroparasitos em escolares da periferia da Universidade Estadual do Maranhão. Pesquisa em Foco, São Luís, v. 16, n. 1, p. 7-14, 2008.

Submetido em: 22/09/2019

Aceito em: 08/02/2021 